\title{
The Effects of Physical Exercise on Plasma levels of Relaxin, NTproANP, AND NTproBNP in PATIENTS with Ischemic HeArT DisEASE
}

\author{
M. Heringlake ${ }^{1 *}$, T. Kox ${ }^{4 *}$, J. Poeling ${ }^{5}$, S. Klaus ${ }^{6}$, T. Hanke' , N. Franz ${ }^{4}$, F. Eberhardt ${ }^{3}$, H. Heinze ${ }^{1}$, \\ F. P. Armbruster ${ }^{7}$ L. Bahlmann ${ }^{8}$
Kliniken für Anästhesiologie ${ }^{1}$, Herzchirurgie ${ }^{2}$, und Kardiologie ${ }^{3}$, Universität zu Lübeck, Lübeck, Germany; Kliniken für Kardiologie ${ }^{4}$ und Herzchirurgie ${ }^{5}$, Schüchtermann-Klinik, Bad Rothenfelde, Germany; Klinik für Anästhesiologie ${ }^{6}$, Herz-Jesu-Krankenhaus, Münster - Hiltrup, Germany; Immundiagnostik $\mathrm{AG}^{7}$, Bensheim, Germany;
Klinik für Anästhesiologie ${ }^{8}$, Krankenhaus Weser-Egge, Höxter, Germany

\begin{abstract}
The insulin-like and vasodilatatory polypeptide relaxin (RLX), formerly known as a pregnancy hormone, has gained interest as a potential humoral mediator in human heart failure. Controversy exists about the relation between plasma levels of RLX and the severity of heart failure. The present study was designed to determine the course of RLX, atrial, and brain natriuretic peptide (NT-proANP and NT-proBNP) during physical exercise in patients with ischemic heart disease (IHD) and to relate hormone levels to peak cardiac power output (CPO) as a measure of cardiopulmonary function with prognostic relevance. 40 patients with IHD were studied during right-heart-catheterization at rest and during supine bicycle ergometry. RLX, NTproBNP, and NTproANP were determined before, during exercise, and after recovery. NT-proANP and NT-proBNP levels increased during maximal charge, and recovery while RLX levels decreased. Cardiac power output at maximal charge correlated inversely with NTproANP and NTproBNP but positively with RLX. Patients with high degree heart failure $(\mathrm{CPO}<1.96 \mathrm{~W})$ had higher NTproANP and NTproBNP and lower RLX levels than patients with low degree heart failure. While confirming the role of NTproANP and NTproBNP as markers for the severity of heart failure, the present data do not support the concept that plasma levels of RLX are related to the severity of myocardial dysfunction and that systemic RLX acts as a compensatory vasodilatatory response hormone in ischemic heart disease.
\end{abstract}

Key words: Heart failure, coronary artery disease, cardiorenal integration, natriuretic peptides, hemodynamics, right heart catheterization.

\section{INTRODUCTION}

The insulin-like polypeptide relaxin (RLX), formerly known as a pregnancy hormone [1], has gained interest as a potential humoral mediator in human heart failure

\footnotetext{
* Both authors contributed equally to this study.
}

[2]. Dschietzig and coworkers [3] have shown that the plasma levels of RLX are increased in relation to the severity of myocardial dysfunction, that the heart is a relevant source of RLX during cardiac failure, and that plasma RLX concentrations are related to left ventricular filling pressures. Comparably, Fisher and coworkers observed high RLX levels in patients with heart failure [4]. In contrast, Kupari and coworkers [5] failed to detect increased plasma RLX levels in patients with aortic valve disease but confirmed that the heart is capable of producing RLX during heart failure states.

Krueger et al. analyzed the effects of physical exercise on plasma RLX levels in patients with heart failure in comparison with healthy controls and observed that plasma RLX levels neither were different between groups nor changed during physical exercise [6]. However, this study investigated a heterogeneous group of heart failure patients including subjects with idiopathic cardiomyopathy as well as patients with ischemic heart disease. Additionally the study did not relate RLX levels to hemodynamics and the plasma concentrations of natriuretic peptides.

The present study was thus designed to determine the effects of physical exercise (bicycle ergometry) on the plasma levels of RLX and N-terminal pro A and B type natriuretic peptides (NTproANP and NTproBNP, respectively) as established humoral markers for the severity of myocardial dysfunction in a homogeneous group of patients with ischemic heart disease of different severity. Additionally, the relationsship between cardiac power output at maximal charge and the respective hormone levels were analyzed.

\section{Materials AND Methods}

The investigation conforms with the principles outlined in the Declaration of Helsinki. Following approval by the institutional review board and written informed consent, 40 medical patients with ischemic heart disease were studied during routine right-heartcatheterization (RHC). None of the female patients was at childbearing age. Demographic data are given in Table 1. Left ventricular ejection fraction was determined echocardiographically before RHC. 
Table 1. Demographics.

\begin{tabular}{ll}
\hline Age (years) & $60(38-78)$ \\
Weight $(\mathrm{kg})$ & $84(63-115)$ \\
Height $(\mathrm{cm})$ & $175(160-190)$ \\
Male/Female & $35 / 5$ \\
LVEF [\%] & $47(20-65)$ \\
& \\
Diabetes mellitus & 7 \\
Smoking (Yes/No) & $18 / 22$ \\
AOD & 4 \\
AH & 32 \\
Previous MI & 28 \\
Previous CABG & 19 \\
Plasma creatinine [mmol*1-1] & $65(52-103)$ \\
\hline
\end{tabular}

Demographic data and plasma creatinine levels of 40 patients undergoing ergometric exercise testing. Data are median \pm range. LVEF: left ventricular ejection fraction (echocardiograpy); AOD: arterial occlusive disease; AH: arterial hypertension; MI: myocardial infarction; CABG: coronary artery bypass grafting.

Patients were studied after overnight fasting. After a 30 min resting period in the supine position a pulmonary artery catheter (Edwards Lifescience, Irvine, USA) was inserted via the brachial vein of the nondominant arm. Cardiac output was determined by bolus thermodilution. The results are given as the average of three measurements. Arterial blood pressure was determined oscillometrically, heart rate was determined by electrocardiography.

The hemodynamic variables measured were: mean arterial pressure (MAP), cardiac output and index (CO and $\mathrm{CI})$, central venous pressure (CVP), systolic, mean, diastolic and occlusion pulmonary artery pressures (PAPS, PAPM, PAPD, PAOP), and mixed venous saturation $\left(\mathrm{SvO}_{2}\right)$. Peak cardiac power output was calculated as CO x MAP x 0.00222 during maximal charge

Pulmonary arterial blood samples for the determination of RLX, NTproANP, and NTproBNP were drawn from the pulmonary artery. Blood was sampled in EDTA-tubes containing $5000 \mathrm{U}$ aprotinine (Trasylol, Bayer, Germany) and immediately spun for 10 minutes at $3400 \mathrm{rpm}$. Supernatants were stored at $-70{ }^{\circ} \mathrm{C}$ for further analysis.

After completing the baseline measurements ( $\mathrm{t} 1$ ), the patients performed an ergometric test in the supine position. Starting with 25 watt, charge was increased in 25 to 100 watt steps every sixth minute according to the estimated capacity of the patient. Repeated measurements were performed during each step test for a maximum of 3 steps $(\mathrm{t} 2, \mathrm{t} 3, \mathrm{t} 4)$. Maximal charge varied from 25 to 200 watts depending on the achievement of stop criteria.

Stop criteria were: achievement of submaximal heart rate (200 bpm minus age), missing increase of heart rate during charge, arterial blood pressure $>240 / 120 \mathrm{mmHg}$, PAOP > $40 \mathrm{mmHg}$, PAPM > 60 $\mathrm{mmHg}, \mathrm{SvO}_{2}<40 \%$, ST-lowering $>0,2 \mathrm{mV}$, a decrease in arterial blood pressure during charge, angina pectoris, shortness of breath, peripheral exhaustion, and recurrent ventricular arrhythmias despite of repositioning the PA-catheter.

Ischemia during ergometric testing was defined as angina pectoris combined with typical electrocardiographic signs of myocardial ischemia and/or a sudden increase in PAOP of more than $20 \mathrm{mmHg}$.

After a resting period of 10 minutes a blood analyses was drawn for determination of recovery hormone levels (t5).

\section{DETERMINATION OF RELAXIN}

RLX was determined by a sandwich-type enzyme immunoassay (EIA) as described previously [7]. Shortly, this assay is based on two specific polyclonal antibodies raised in rabbits against recombinant human gene H2-RLX. The detection limit of this assay is 1.2 $\mathrm{pg}^{*} \mathrm{ml}-1$. The standard range is 1.2 to $250 \mathrm{pg}^{*} \mathrm{ml}^{-1}$. Recovery in plasma is $97 \pm 4.5 \%$. The intra-assay coefficient of variance at $15.3 \mathrm{pg} * \mathrm{ml}^{-1}$ was $9.6 \%(\mathrm{n}=18)$, the inter-assay coefficient of variance at $15.6 \mathrm{pg}^{*} \mathrm{ml}^{-1}$ was $10.2 \%(\mathrm{n}=12)$. The assay has a cross-reactivity of less than $0.01 \%$ with insulin, insulin-like growth factor, $\mathrm{LH}$, and $\mathrm{FSH}$.

\section{DETERMINATION OF NT PROANP}

NTproANP was determined by a competitive-binding radioimmunoassay with magnetic solid phase technique in a modification of Sundsfjord et al. [8] using the same rabbit-anti-rat proANP polyclonal serum, human proANP (1-30) from Peninsula Lab (Bachem Ltd, St. Helene, UK) as the standard, and iodined proANP 1-30 purified by HPLC for radiolabeling. In order to achive high sensitivity and good precision, Dynabeads M280 with sheep-anti-rabbit IgG (Dynal Biotech,Oslo, Norway) as solid phase and second antibody were used. The coefficient of variance at 425 , 1163 , and $2490 \mathrm{pmol}^{*} 1^{-1}$ was $7.5,3.7$, and $3.4 \%$, respectively. The detection limit was $30 \mathrm{pmol} / 1$.

\section{DETERMINATION OF NT PROBNP}

NTproBNP was determined by an electrochemoluminescence immunoassay (Elecsys proBNP sandwich immunoassay; Roche Diagnostics, Basel, Switzerland) on Elecsys 2010 [9]. The mean intraassay variance was $4.3 \%$ (range: 2.7 to $5.9 \%$ for plasma samples with a concentration between 7.6 to $2732 \mathrm{pmol}^{*} \mathrm{1}^{-1}$ with an interassay variance of $3.2 \%$ ). The lower detection limit was $0.6 \mathrm{pmol}^{*} \mathrm{1}^{-1}$.

\section{Statistical Analyses}

Following the observation that only 23 of the patients were capable of performing all exercise steps and that 8 patients achieved the stop criteria already during exercise step 1, peak charge levels for hemodynamic and hormonal parameters were used for further analysis to achieve comparability between individuals.

After the ergometric testing, patients were grouped according to peak cardiac power output. As suggested by Williams and coworkers [10], a cut-off value $<1.96$ 
watt was used to divide the patients in groups of high degree (HDHF: $\mathrm{n}=18$ ) and low degree heart failure (LDHF: $\mathrm{n}=22$ ).

Data are given as median \pm range. With respect to the high variability of baseline hormone levels, data were analyzed non-parametrically. Friedman' test followed by Wilcoxon's matched pairs test was used for intra-individual comparisons. Mann-Whitney $U$ test was applied for analyses of differences between the HDHF and the LDHF group. Fisher' s exact test was used to analyze between group differences of nominal variables. Correlation analyses were performed by Spearman's rank correlation test. A p-value of less than 0.05 indicates statistical significance; $\mathrm{p}=$ n.s. denotes no statistical significance.

\section{RESULTS}

All patients underwent successful ergometric testing. No complications related to the right heart catheterization occurred. Five patients in the HDHF group but none in LDHF were tested postive for myocardial ischemia $(p=0.013)$.

\section{HEMODYNAMICS}

The course of hemodynamics is given in Table 2, showing that exercise was associated with profound changes in cardiac index, mean arterial pressure, mixed venous saturation and filling pressures. Peak cardiac power output (CPO) at the individual maximal charge was $2.7(1.2-5.5) \mathrm{W}$.

Analysis of between group differences in the HDHF and the LDHF groups revealed that the
HDHF group (peak CPO: 1.46 (1.04-1.86) W) had higher baseline pulmonary artery pressures (data not shown) and PAOP levels $(10$ (4-28) vs. 6 (4-14) $\mathrm{mmHg}, \mathrm{p}=0.03)$, a lower baseline $\mathrm{SvO}_{2}$ (63 (58-73) vs. $69(63-75) \%, p=0.002)$, higher peak exercise PAOP (31 (13-43) vs. $23(7-35) \mathrm{mmHg}, \mathrm{p}=0.005)$ but lower MAP (111 (98-126) vs. 122 (100-142) mmHg, p $=0.02)$ and CI (3.6 (2.7-4.8) vs. 6.3 (4.3-9.4), p $<0.0001)$ than the LDHF group.

Differences in demographics and medication in both groups is given in Table 3, showing that patients in the HDHF group were more frequently tested positively for ischemia during exercise and were more frequently treated with digitalis.

\section{HORMONES}

The course of NTproANP, NTproBNP and RLX in all patients is given in Figure 1, showing that both natriuretic peptides increased modestly during maximal charge and recovery and that RLX levels during recovery were significantly lower than baseline levels.

The course of hormones in the HDHF and the LDHF groups is depicted in Figure 2, showing that plasma NTproANP and NTproBNP levels were higher and RLX levels were lower in the HDHF in comparison with the LDHF group. NTproANP and NTproBNP in the EDHF and the LDHF increased comparably to the changes observed in all patients (Fig. 2) while no significant changes in plasma RLX were observed.

Individual changes in RLX levels in the HDHF and LDHF group are given in Figure 3 a. and 3.b, showing a high variability at baseline and heterogenous re-

Table 2. Hemodynamics.

\begin{tabular}{|c|c|c|c|c|}
\hline & t1 & $\mathrm{t} 2$ & t3 & $\mathrm{t} 4$ \\
\hline $\mathrm{n}$ & 40 & 40 & 32 & 23 \\
\hline $\begin{array}{l}\text { charge } \\
{[\mathrm{W}]}\end{array}$ & & $\begin{array}{l}25 \\
(25-25)\end{array}$ & $\begin{array}{l}50 \\
(50-100)\end{array}$ & $\begin{array}{l}100 \\
(75-200)\end{array}$ \\
\hline $\begin{array}{l}\text { CI } \\
{\left[1 / \mathrm{min} / \mathrm{m}^{2}\right]}\end{array}$ & $\begin{array}{l}2.9 \\
(1.4-4.4)\end{array}$ & $\begin{array}{l}3.4^{*} \\
(2.5-5.3)\end{array}$ & $\begin{array}{l}4.6^{*} \\
(3.1-7.2)\end{array}$ & $\begin{array}{l}6.3^{*} \\
(4.3-9.4)\end{array}$ \\
\hline $\begin{array}{l}\text { MAP } \\
{[\mathrm{mmHg}]}\end{array}$ & $\begin{array}{l}92 \\
(75-126)\end{array}$ & $\begin{array}{l}112^{*} \\
(83-116)\end{array}$ & $\begin{array}{l}112^{*} \\
(95-114)\end{array}$ & $\begin{array}{l}121^{*} \\
(100-140)\end{array}$ \\
\hline $\begin{array}{l}\text { HR } \\
{[\mathrm{bpm}]}\end{array}$ & $\begin{array}{l}68 \\
(44-95)\end{array}$ & $\begin{array}{l}88^{*} \\
(62-114)\end{array}$ & $\begin{array}{l}105^{*} \\
(70-134)\end{array}$ & $\begin{array}{l}122^{*} \\
(102-173)\end{array}$ \\
\hline $\begin{array}{l}\mathrm{SvO}_{2} \\
{[\%]}\end{array}$ & $\begin{array}{l}66 \\
(58-75)\end{array}$ & $\begin{array}{l}41 * \\
(27-58)\end{array}$ & $\begin{array}{l}36^{*} \\
(21-55)\end{array}$ & $\begin{array}{l}32^{*} \\
(20-49)\end{array}$ \\
\hline $\begin{array}{l}\text { CVP } \\
{[\mathrm{mmHg}]}\end{array}$ & $\begin{array}{l}4 \\
(2-12)\end{array}$ & $\begin{array}{l}16 \\
(7-26)\end{array}$ & $\begin{array}{l}9 \\
(8-11)\end{array}$ & $\begin{array}{l}9^{*} \\
(4-15)\end{array}$ \\
\hline $\begin{array}{l}\text { PAPM } \\
{[\mathrm{mmHg}]}\end{array}$ & $\begin{array}{l}15 \\
(10-36)\end{array}$ & $\begin{array}{l}34 * \\
(15-65)\end{array}$ & $\begin{array}{l}36^{*} \\
(21-52)\end{array}$ & $\begin{array}{l}36^{*} \\
(22-56)\end{array}$ \\
\hline $\begin{array}{l}\text { PAOP } \\
{[\mathrm{mmHg}]}\end{array}$ & $\begin{array}{l}8 \\
(4-28)\end{array}$ & $\begin{array}{l}20^{*} \\
(9-43)\end{array}$ & $\begin{array}{l}20^{*} \\
(8-35)\end{array}$ & $\begin{array}{l}23^{*} \\
(7-39)\end{array}$ \\
\hline
\end{tabular}

The course of hemodynamics in 40 patients with ischemic heart disease undergoing ergometric bicycle testing. t1: Baseline after 30 min rest; t2 to t4: ergometric testing with increasing charges. CI: cardiac index; MAP: mean arterial blood pressure, HR: heart rate; $\mathrm{SvO}_{2}$ : mixed venous oxygen saturation; CVP: central venous pressure; PAPM: mean pulmonary artery pressure; PAOP: pulmonary artery occlusion pressure. *: significant difference $(p<0.05)$ in comparison with t1 (Friedman's test followed by Wilcoxon's matched pairs test). 
Table 3. Demographic data and medication in the LDHF and HDHF groups.

\begin{tabular}{|c|c|c|c|c|}
\hline & & LDHF $(n=22)$ & HDHF (n = 18) & sign. \\
\hline Age (years) & & $59(38-64)$ & $60(39-78)$ & n.s. \\
\hline Weight (kg) & & $86(70-115)$ & $82(63-91)$ & n.s. \\
\hline Height (cm) & & $180(160-190)$ & $172(160-185)$ & n.s. \\
\hline Male/Female & & $21 / 1$ & $14 / 4$ & n.s. \\
\hline LVEF [\%] & & $60(20-65)$ & $45(30-60)$ & n.s. \\
\hline Previous MI & [yes/no] & $18 / 4$ & $10 / 8$ & n.s. \\
\hline Previous CABG & [yes/no] & $10 / 22$ & $9 / 18$ & n.s \\
\hline Previous PCI & [yes/no] & $11 / 22$ & $10 / 18$ & n.s. \\
\hline Pos. ischemia testing & [yes/no] & $0 / 22$ & $5 / 13$ & $\mathrm{p}=0.013$ \\
\hline Duration of IHD & [months] & $1(1-50)$ & $2(1-120)$ & n.s. \\
\hline Betablocker & [yes/no] & $22 / 0$ & $18 / 0$ & n.s. \\
\hline ACE-inhibitor/ARB & [yes/no] & $17 / 5$ & $17 / 1$ & n.s. \\
\hline Diuretics & [yes/no] & $5 / 17$ & $9 / 9$ & $(p=0.08)$ \\
\hline Aldosterone-Blocker & [yes/no] & $0 / 22$ & $1 / 17$ & n.s. \\
\hline Digitalis & [yes/no] & $2 / 20$ & $7 / 11$ & $\mathrm{p}=0.025$ \\
\hline
\end{tabular}

Demographic data and medication of patients with ischemic heart disease (IHD) grouped according to the results of ergometric exercise testing in a high degree heart failure (HDHF) and a low degree heart failure group (LDHF). Data are median \pm range. LVEF: left ventricular ejection fraction (echocardiograpy); MI: myocardial infarction; CABG: coronary artery bypass grafting; PCI: percutaneus coronary intervention; positive ischemia testing: please see text for details; ARB: angiotensin receptor blocker. $\mathrm{p}<0.05$ : significant between group difference (continuous data: Mann-Whitney U, nominal data: Fisher's exact test).

sponses during exercise and recovery in both groups. From baseline to recovery RLX increased in 8 and decreased in 10 patients in the HDHF-group and increased in 14 but decreased in 8 patients in the LDHFgroup.

\section{Correlation Analyses}

Correlation analyses revealed no correlation between RLX baseline plasma levels and corresponding hemodynamic indices and the EF. Inverse correlations between RLX levels and NTproANP (Spearman's rho $(\mathrm{r} / \mathrm{R})=-0.33, \mathrm{p}=0.047)$ and NTproBNP $(\mathrm{r} / \mathrm{R}=$ $-0.41, \mathrm{p}=0.013)$ were observed. The correlation between RLX baseline levels and peak CPO was $\mathrm{r} / \mathrm{R}=$ $0.48(\mathrm{p}=0.0056)$.

In contrast, NTproANP and NTproBNP baseline levels were strongly to moderately associated with hemodynamic parameters at rest (NTproANP vs: EF: $r / R=-0.36(p=0.02)$; PAPS: $r / R=0.72(p<0.0001)$; PAPD: $\mathrm{r} / \mathrm{R}=0.67(\mathrm{p}<0.0001)$ PAPM: $\mathrm{r} / \mathrm{R}=0.66(\mathrm{p}$ $<0.0001)$; PAOP: $\mathrm{r} / \mathrm{R}=0.71(\mathrm{p}<0.0001) ; \mathrm{SvO}_{2}$ : $\mathrm{r} / \mathrm{R}$ $=-0.47(\mathrm{p}=0.0045) ;$ NTproBNP vs: $\mathrm{EF}: \mathrm{r} / \mathrm{R}=-0.52$ ( $\mathrm{p}=0.008)$; PAPS: $\mathrm{r} / \mathrm{R}=0.53(\mathrm{p}=0.0016)$; PAPD: $\mathrm{r} / \mathrm{R}=0.48(\mathrm{p}=0.004) ;$ PAPM: $\mathrm{r} / \mathrm{R}=0.48(\mathrm{p}=$ 0.0038); PAOP: $\mathrm{r} / \mathrm{R}=0.51(\mathrm{p}=0.0021) ; \mathrm{SvO}_{2}: \mathrm{r} / \mathrm{R}=$ $-0.38(\mathrm{p}=0.02))$. The correlation between NTproANP and NTproBNP baseline levels and peak CPO was $r / R=-0.55(p=0.0045)$ and $r / R=-0.66(p$ $<0.0001)$, respectively.

Only minor correlations were observed between hemodynamics changes from baseline to maximal charge and changes in RLX and NTproANP (delta MAP vs. delta RLX: $\mathrm{r} / \mathrm{R}=0.35$ ( $\mathrm{p}=0.046)$; delta NTproANP vs. delta PAOP: $\mathrm{r} / \mathrm{R}=0.39(\mathrm{p}=0.0025)$; delta NTproANP vs. delta SVI: $\mathrm{r} / \mathrm{R}=-0.35$ ( $\mathrm{p}=0.044)$; delta NTproANP vs. delta proBNP: $\mathrm{r} / \mathrm{R}=0.39(\mathrm{p}=$ 0.027)).

\section{DISCUSSION}

Recently, Dschietzig el al. suggested that the polypeptide RLX is "an important player in heart failure" [3]. This assumption was based on the observation that the heart is a RLX net producer in heart failure states and that the plasma levels of RLX in a study in patients presenting with acute cardiac decompensation were closely related to left ventricular filling pressures and the clinical severity of myocardial dysfunction. Additionally this group showed that RLX is a functional antagonist of the vasoconstrictory effects of endothelin-1; suggesting that the increase of RLX in heart failure may be a compensatory response [2]. However, data from other groups on the role of RLX in heart failure are controversial. Fisher and coworkers reported that increased plasma RLX levels in heart failure patients have no prognostic relevance and failed to detect a meaningful correlation between this hormone an NTproBNP as an accepted humoral marker of myocardial dysfunction [4]. Comparably, Kupari and coworkers did not detect any correlation between plasma RLX levels and "any measure of cardiac structure or function" in a group of patients with isolated aortic valve disease [5], and Krüger et al. [6] did not observe any difference in RLX levels at baseline as well as at peak exercise in compensated heart failure patients compared to a healthy control group. Thus obviousy the RLX impact in the setting of human heart failure has not been satisfactorily solved.

Right heart catherization with ergometric testing is an established method for the quantification of cardiac dysfunction under different loading conditions. 

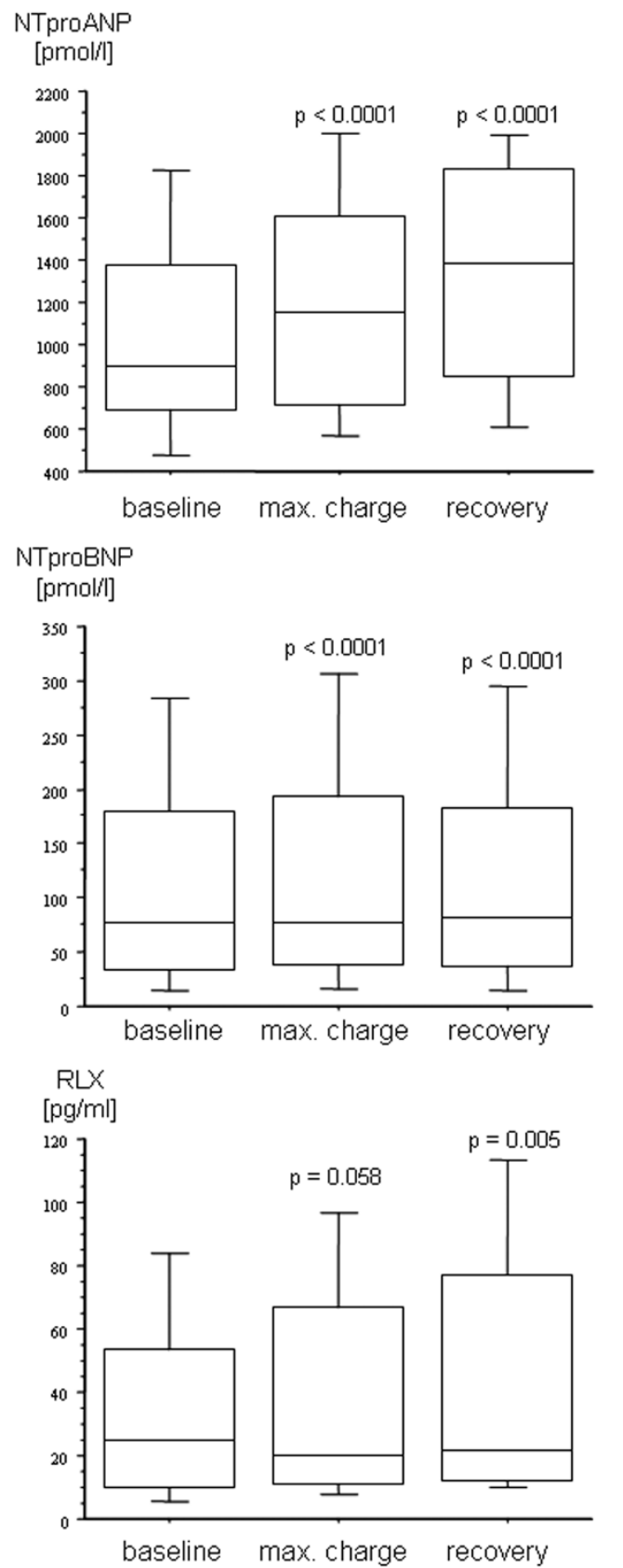

Fig. 1. The course of the plasma levels of the N-terminal prohormones of the A- and B-type natriuretic peptides (NTproANP, NTproBNP) and relaxin (RLX) in patients with ischemic heart disease undergoing physical exercise testing. Baseline: after 30 min supine rest; max. charge: during peak exercise; recovery: $10 \mathrm{~min}$ after peak exercise. Data are given as box blots. P-values denote a significant difference versus baseline (Friedman's test followed by Wilcoxon's matched pairs test).

Besides conventional hemodynamic parameters (cardiac index and filling pressures) that may be used for quantification of cardiac function, peak cardiac power output - the product of cardiac output and mean arterial blood pressure - is a measure of myocardial dysfunction of prognostic relevance [10] which integrates cardiac output and ventricular afterload [11].

The results of our study question the role of plasmatic RLX as a compensatory humoral mediator in
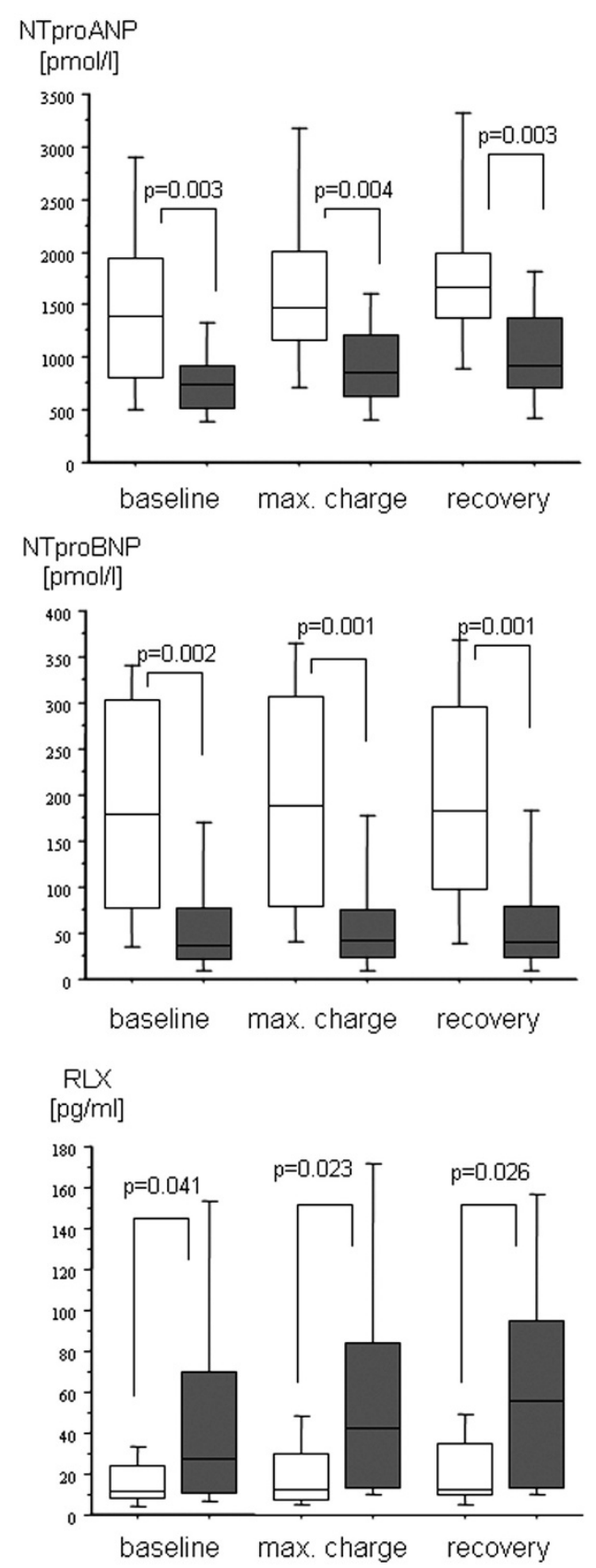

Fig. 2. The course of the plasma levels of the N-terminal prohormones of the A- and B-type natriuretic peptides (NTproANP, NTproBNP) and relaxin (RLX) in patients with ischemic heart disease undergoing ergometric exercise testing grouped according to peak cardiac power output (CPO) less or greater than $1.96 \mathrm{~W}$. Baseline: after $30 \mathrm{~min}$ supine rest; max. charge: during peak exercise; recovery: $10 \mathrm{~min}$ after peak exercise. High degree heart failure (open box plots: peak $\mathrm{CPO}<1.96 \mathrm{~W} ; \mathrm{n}=18$ ); low degree heart failure (grey box plots: $\mathrm{CPO}>1.96 \mathrm{~W} ; \mathrm{n}=22$ ). P-values denote significant differences between groups (Mann-Whitney - U test). Friedman's test followed by Wilcoxon's matched pairs test revealed significant within group changes in NTproANP and NTproBNP levels and no significant changes in plasma RLX.

human heart failure: We neither did observe any direct correlation between plasma RLX and hemodynamic function at rest nor a positive correlation with NTproANP or NTproBNP baseline levels. In contrast, 
RLX

$[\mathrm{pg} / \mathrm{ml}]$

a.

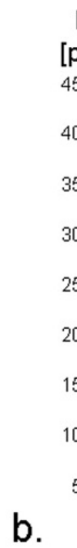

[pg/

$\mathrm{RLX}$ $[\mathrm{pg} / \mathrm{ml}]$

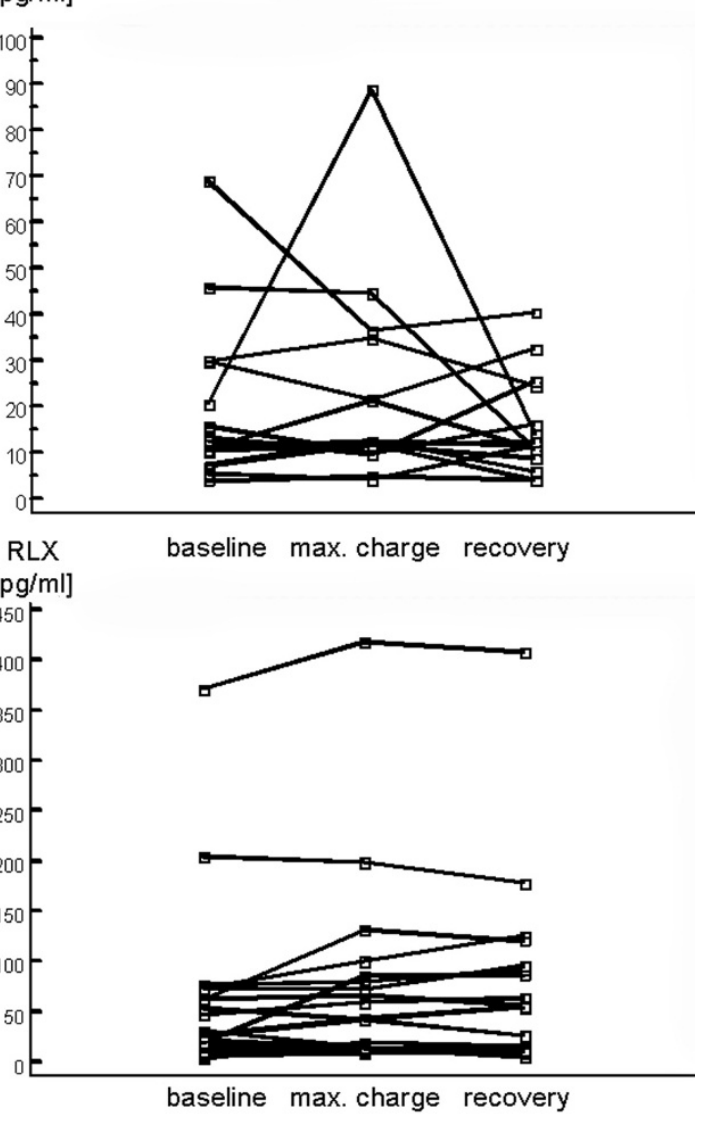

Fig. 3. The individual changes in plasma relaxin (RLX) levels in patients with ischemic heart disease undergoing ergometric exercise testing grouped according to peak cardiac power output (CPO) less or greater than $1.96 \mathrm{~W}$. Baseline: after $30 \mathrm{~min}$ supine rest; max. charge: during peak exercise; recovery: 10 min after peak exercise. a. high degree heart failure (peak $\mathrm{CPO}<1.96 \mathrm{~W} ; \mathrm{n}=18$ ); b. low degree heart failure (CPO $>1.96 \mathrm{~W} ; \mathrm{n}=22$ ). Friedman's test followed by Wilcoxon's matched pairs test revealed no significant within group changes. Please note the different scales on the y-axes.

inverse correlations between RLX levels and natriuretic peptides at baseline and a positive correlation between RLX and peak CPO were detected. Additionally, RLX levels tended to decrease during exercise and decreased during recovery during exercise (in the whole group), showed a highly variable and inhomogeneous response pattern in the HDHF and the LDHF group, and were lower in patients with more severe myocardial dysfunction. It is of note that we have recently shown that plasma RLX levels in healthy volunteers were higher than those reported by Dschietzig and coworkers for patients with severe myocardial failure [7].

It remains to be determined how the discrepancies between the above mentioned studies can be explained; even taking into account that Dschietzigs patients were in acute heart failure while the patients in the other studies were performed in stable patients with either chronic heart failure $[4,5,6]$ or as in the present study in patients with ischemic heart disease and varying degree of myocardial dysfunction. Inter- estingly, patients in the HDHF group were more frequently tested positive for ischemia during exercise. The neurohumoral response in these patients may thus - at least in part - be more intensively influenced by myocardial ischemia than by myocardial failure. However, plasma levels of RLX were not significantly different between patients with or without ischemia during the study period (data not shown). Comparably, patients in the HDHF were more frequently treated with digitalis and as a trend $(\mathrm{p}=0.08)$ more frequently with diuretics. Since no data are available on the effects of acute myocardial ischemia, digitalis, and diuretics on plasma levels of RLX any attempt to explain the findings of the present study on the basis of these differences remains speculative.

It is of note that the immunoassay kit in all studies mentioned so far was purchased from the same manufacturer. In contrast to Dschietzig and coworkers (personal communication), we added aprotinine during blood sampling to prevent protein degradation. This, however, may explain absolut differences in RLX levels between both studies but does not help to explain the lack of relative differences, i.e the fact that patients with more severe myocardial dysfunction had lower RLX levels. Consequently, variations in laboratory technology are not helpful in explaining the contradictory results between Dschietzigs [3] and our findings. It is of note that the analyses in the present study were performed in the reference laboratory of the manufacturer (Immundiagnostik AG), rendering possible errors in the analytical technique rather unlikely.

With respect to the pleiotropic effects of RLX, several alternative explanations for these discordant results may be conceivable. For example, plasma RLX levels have been shown to be related to insulin sensitivity and serum fibrinogen levels in female patients with type 2 diabetes mellitus [12, 13], suggesting, that this "“insulin-like" hormone is indeed related to changes in metabolism.

Additionally, a lack of systemic changes in RLX levels does not exclude effects of this hormone on the organ or cellular levels. This may be derived from the observation that acute volume loading in healthy volunteers leads to a significant increase in urinary excretion of RLX despite unchanged plasma RLX levels [7]. Variable results have been reported on the role of RLX as a paracrine humoral mediator in the cardiovascular system. In a very recent publication, Dschietzig an coworkers have shown upregulation of RLX mRNA-transcription and RLX protein content in the left atria and ventricles of spontaneous hypertensive rats in comparison with nonhypertensive Wistar Kyoto rats and a close inverse correlation between left atrial and ventricular relative myocardial mass and the RLX protein content [14], suggesting that this peptide may exert antihypertrophic effects in this model of increased vascular resistance. It is not known, if RLX may have comparable effects in humans.

In contrast to RLX, NTproANP and NTproBNP were highly accurate in differentiating between patients with less and more severe myocardial dysfunction, either in the resting state as well as during exercise. This supports the well established role of these hormones for the detection of myocardial failure [9]. 
Few data is available about the course of NTproBNP during physical exercise and no data exists about NTproANP. Scharhag et al. observed that NTproBNP increased after a walking test in patients with IHD and that patients with more severe myocardial dysfunction showed a more pronounced increase of this hormone 30 min after exercise. In contrast to our data, no correlation between NTproBNP baseline levels and peak cardiac power output was observed. [15]. The changes in NTproANP levels were less pronounced as it has been shown for the active compound ANP [16]. This may be explained by the different plasma half-lifes and the higher plasma levels of the N-terminal prohormones in comparison with the active hormones.

In summary, the data presented in this study clearly shows that patients with ischemic heart disease and myocardial dysfunction - diagnosed by invasive hemodynamic parameters - present with lower levels of plasma relaxin than patients with preserved myocardial function and that plasma RLX levels are inversely related to the severity of heart disease in these patients. Therefore, in our opinion, the significance of RLX as an important compensatory hormone in IHD patients has not been clearly answered so far.

Acknowledgements: We acknowledge the help of Charlotte Neubert from Immundiagnostik AG, Bensheim, Germany and Ragnhild Wergeland, MD, Institute of Clinical Biochemistry, Rikshospitalet, University of Oslo, Norway for performing the hormone analyses. We thank Roche Diagnostics, Germany, for providing the kits for determination of NTproANP and NTproBNP. We also appreciate the great amount of effort of our institutional statistician Michael Hüppe, $\mathrm{PhD}$, when supporting our study.

Declaration of a potential conflict of interest: The authors Matthias Heringlake and Frank Eberhardt have received scientific support by Roche Diagnostics, Germany. Franz-Paul Armbruster is the director of Immundiagnostik AG, the manufacturer of the relaxin assay.

\section{REFERENCES}

1. Conrad KP, Nowak J. Emerging role of relaxin in renal and cardiovascular function. Am J Physiol Regul Integr Comp Physiol 287: 250-261, 2004.

2. Fisher C, MacLean M, Morecroft I, Seed A, Johnston F, Hillier C, McMurray J. Is the pregnancy hormone relaxin also a vasodilator peptide secreted by the heart? Circulation 106:292-295, 2002.

3. Dschietzig T, Richter C, Bartsch C, Laule M, Armbruster FP, Baumann G, Stangl K. The pregnancy hormone relaxin is a player in human heart failure. FASEB J 15:21872195, 2001.

4. Fisher C, Berry C, Blue L, Morton JJ, McMurray J. N-terminal pro $B$ type natriuretic peptide, but not the new putative cardiac hormone relaxin, predicts prognosis in $\mathrm{pa}$ tients with chronic heart failure. Heart 89: 879-881, 2003.

5. Kupari M, Mikkola TS, Turto H, Lommi J. Is the pregnancy hormone relaxin an important player in human heart failure? Eur J Heart Fail 7: 195-198, 2005.
6. Krüger S, Graf J, Merx MW, Stickel T, Kunz D, Hanrath $\mathrm{P}$, Janssens U. Relaxin kinetics during dynamic exercise in patients with chronic heart failure. Eur J Intern Med 15: 54-56, 2004.

7. Heringlake M, Heide C, Bahlmann L, Eichler W, Pagel H, Schmucker P, Wergeland R, Armbruster FP, Klaus S. Effects of tilting and volume loading on plasma levels and urinary excretion of relaxin, NT-pro-ANP, and NT-proBNP in male volunteers. J Appl Physiol 97:173-179, 2004.

8. Sundsfjord JA, Thibault G, Larochelle P, Cantin M. Identification and plasma concentrations of the $\mathrm{N}$-terminal fragment of proatrial natriuretic factor in man. J Clin Endocrinol Metab 66: 605-610, 1988.

9. Mueller T, Gegenhuber A, Poelz W, Haltmayer M. Comparison of the Biomedica NT-proBNP enzyme immunoassay and the Roche NTproBNP chemiluminescence immunoassay: implications for the prediction of symptomatic and asymptomatic structural heart disease. Clin Chem 49: 976-979, 2003.

10. Williams SG, Cooke GA, Wright DJ, Parsons WJ, Riley RL, Marshall P, Tan LB. Peak exercise cardiac power output - A direct indicator of cardiac function strongly predictive of prognosis in chronic heart failure. Eur Heart J 22: 1496-1503, 2001.

11. Bromley PD, Hodges LD, Brodie DA. Physiological range of peak cardiac power output in healthy adults. Clin Physiol Funct Imaging 26: 240-246, 2006.

12. Szepietowska B, Gorska M, Szelachowska M. Plasma relaxin concentration is related to beta-cell function and insulin sensitivity in women with type 2 diabetes mellitus. Diabetes Res Clin Pract 79: 1-3, 2008.

13. Schöndorf T, Lübben G, Hoopmann M, Borchert M, Forst T, Hohberg C, Löbig M, Armbruster FP, Roth W, Grabellus M, Pfützner A. Relaxin expression correlates significantly with serum fibrinogen variation in response to antidiabetic treatment in women with type 2 diabetes mellitus. Gynecol Endocrinol 23: 356-60, 2007.

14. Dschietzig T, Bartsch C, Kinkel T, Baumann G, Stangl K. Myocardial relaxin counteracts hypertrophy in hypertensive rats. Ann N Y Acad Sci 1041: 441-443, 2005.

15. Scharhag J, Herrmann M, Weissinger M, Herrmann, W, Kindermann W. N-terminal B-type natriuretic peptide concentrations are similarly increased by 30 minutes of moderate and brisk walking in patients with coronary artery disease. Clin Res Cardiol 96: 218-226, 2007.

16. Kato M, Kinugawa T, Omodani H, Osaki S, Ogino K, Hisatome I, Miyakoda H, Fujimoto Y. Augmented response in plasma atrial natriuretic peptide to dynamic exercise in patients with congestive heart failure. Jpn Circ J 60: 909-916, 1996.

Received: January 23, 2009 / Accepted: February 9, 2009

Address for correspondence:

Priv.-Doz. Dr. Matthias Heringlake

Klinik für Anästhesiologie

Universität zu Lübeck

Ratzeburger Allee 160

23538 Lübeck

Germany.

Phone: $\quad++49 / 451 / 500-4057$

Fax: $\quad++49 / 451 / 500-3405$

E-mail: Heringlake@t-online.de 